\title{
Excluding Lynch syndrome in a female patient with metachronous DNA mismatch repair deficient colon- and ovarian cancer
}

\author{
Stijn Crobach ${ }^{1,6}$ • Anne M. L. Jansen ${ }^{1}$ Marjolein J. L. Ligtenberg ${ }^{2}$. \\ Marije Koopmans ${ }^{3} \cdot$ Maartje Nielsen $^{3} \cdot$ Frederik J. Hes $^{3} \cdot$ Juul T. Wijnen $^{4}$. \\ Winand N. M. Dinjens ${ }^{5} \cdot$ Tom van Wezel ${ }^{1} \cdot$ Hans Morreau ${ }^{1}$
}

Published online: 9 November 2017

(C) The Author(s) 2017. This article is an open access publication

\begin{abstract}
Patients synchronously or metachronously presenting with ovarian and colon cancer can pose diagnostic challenges. A primary colon carcinoma can metastasize to one or both ovaries, two independent primary tumors can arise or an ovarian carcinoma can metastasize to the colon. Clinical and immunohistochemical characterization can aid the diagnosis. Recently, we reported that in difficult cases finding pathogenic $A P C$ variants supports a colonic origin.

In this case report we describe the clinical history of a female patient suspected for Lynch syndrome. She was diagnosed with a bilateral ovarian cancer at age 44 , followed by the detection of a colon carcinoma 12.5 months later. Lesions of both sites showed a DNA mismatch repair deficiency with immunohistochemical loss of MLH1 and PMS2 expression without $\mathrm{MLH1}$ promoter hypermethylation. In absence of germline MMR gene variants identical somatic $M L H 1$ and $C T N N B 1$ gene variants were found, indicating a clonal relation. MMR germline mosaicism was made
\end{abstract}

Stijn Crobach

a.s.1.p.crobach@lumc.nl

1 Department of Pathology, Leiden University Medical Center, Leiden, The Netherlands

2 Department of Human Genetics and Department of Pathology, Radboud University Medical Center, Nijmegen, The Netherlands

3 Department of Clinical Genetics, Leiden University Medical Center, Leiden, The Netherlands

4 Department of Human Genetics, Leiden University Medical Center, Leiden, The Netherlands

5 Department of Pathology, Erasmus MC Cancer Institute, Rotterdam, The Netherlands

6 Department of Pathology, Leiden University Medical Centre, Zone L1-Q, P.O. Box 9600, 2300 RC Leiden, The Netherlands unlikely by ultra deep sequencing of the $M L H 1$ variant in DNA isolated from normal mucosa, blood, urine and saliva. Although initially being suspect for Lynch syndrome it was eventually concluded that a metachronously diagnosed colon carcinoma that metastasized to both ovaries was most likely.

Keywords Lynch syndrome · Ovarian cancer · Colon cancer

\section{Introduction}

In this report we describe a female patient diagnosed with bilateral endometrioid carcinoma of the ovaries at the age of 44. One year later an adenocarcinoma of the colon was detected. The discovery of the colon carcinoma created doubt about the primary origin of the ovarian tumors. Besides, because the patient met the Amsterdam/Bethesda revised criteria, Lynch syndrome (LS) was suggested.

The ovaries can be affected by metastases from several primary tumor sites [1]. Most metastases originate from the gastrointestinal tract, with the colon as most frequent primary location. However, primary ovarian tumors are more common than ovarian metastases; 85 versus $15 \%$ [2]. Since subtypes of primary ovarian cancers (especially endometrioid and mucinous adenocarcinomas) can show overlapping histological and immunohistochemical features with gastrointestinal tumor metastases, it can be difficult to discriminate these [3, 4]. A combined analysis of clinical and molecular features can be helpful in correctly diagnosing these tumors. Reanalyis of this case revealed both macroscopic and microscopic evidence for a colonic origin of the ovarian tumors. This thought was supported by up-to-date extensive molecular analyses that showed a clonal relationship between both 
tumors. Lynch syndrome, including DNA mismatch repair gene mosaicism, was ruled out.

\section{Materials and methods}

\section{Immunohistochemistry}

Immunohistochemistry was performed as previously described [5]. The antibodies and dilutions that were used are as follows: MSH2 (1:25; DAKO Santa Clara, United States), MSH6 (1:400; GeneTex Irvine, United States), PMS2 (1:80; DAKO Santa Clara, United States) and MLH1 (1:40; DAKO Santa Clara, United States), CDX2 (1:80; DAKO Santa Clara, United States), keratin-7 (1:400; DAKO Santa Clara, United States), keratin-20 (1:200; DAKO Santa Clara, United States), ER (1:40; DAKO Santa Clara, United States), PR (1:400; DAKO Santa Clara, United States) and vimentin (1:1000; DAKO Santa Clara, United States).

\section{Methylation specific assay}

The promoter region of $M L H 1$ was analyzed by methylationspecific multiplex ligation-dependent probe amplification (MS-MLPA) as previously described [6].

\section{Microsatellite instability (MSI) analysis}

Microsatellite analysis was performed using five mononucleotide microsatellite markers as previously described [7].

\section{Germline analysis}

Germline analysis of $M L H 1, P M S 2, M H S 2$ and MSH6 variant was performed on DNA isolated from lymphocytes from a blood sample using standard procedures including the analysis for large deletions/duplications by the multiplex ligation-dependent probe amplification (MRC Holland, the Netherlands).

\section{Somatic and mosaicism analysis}

Somatic mutation analysis of $M L H 1$ was performed using a laboratory developed multiplex AmpliSeq based NGS protocol followed by confirmation of detected mutations by Sanger sequencing.

Additional analysis of somatic variations was performed on DNA isolated using a fully automated DNA extraction procedure. The concentration of DNA was measured using a fluorometer (Qubit dsDNA HS, Thermo Fischer Scientific, Waltman MA USA). The amplicon library for targeted sequencing was constructed using AmpliSeq Cancer Hotspot Panel v2. This panel consists of a single primer pool and is designed to detect somatic cancer hotspot pathogenic variants in 207 amplicons covering 50 cancer related genes, including genes as APC, KRAS, TP53, SMAD4 that are often altered in colorectal cancer. The whole $A P C$ gene was analyzed in a separate analysis as in the cancer hotspot panel only the mutation cluster region of $A P C$ is covered. Mosaicism analysis of the identified $M L H 1$ variant was performed by using a panel covering $M S H 2, M S H 6, P M S 2$, MLH1, POLDI and POLE. Libraries were prepared with $10 \mathrm{ng}$ of genomic DNA, and each sample was uniquely barcoded using IonXpress barcodes (Life Technologies). Nextgeneration sequencing was carried out according to the Ion Proton protocol.

\section{Bioinformatic analysis}

The unaligned BAM file generated by the Proton sequencer were mapped against the human reference genome (GRCh37/hg 19) using the TMAP 5.0.7 software with default parameters (https://github.com/iontorrent/TS). Subsequently variant calling was done using the Ion Torrent specific caller, Torrent Variant Caller (TVC)-5.0.2, using the recommended Variant Caller Parameter for Cancer Hotspot Panel v2. Variant interpretation was done using Geneticist Assistant (Softgenetics) which assigns Functional Prediction, Conservation scores and Disease associated information to each variant (http://softgenetics.com/GeneticistAssistant_2.php). Once pathogenicity is assigned to a variant, the same pathogenicity is automatically attributed the next time the variant is observed. Integrative Genomics Viewer (IGV) was used for visually inspecting variants (doi: 10.1093/bib/bbs017). The analysis of the complete $A P C$ gene was performed as described previously [8]. LOH was analyzed by comparison of variant and wild type DNA reads of the NGS results.

\section{Case report}

We describe a female patient with a family history of ovarian cancer (one sister at the age of 56 years), breast cancer (one sister at the age of 59 years), colon cancer (patient's mother at the age of 80 years) and a (non melanoma) skin cancer (the sister diagnosed with breast cancer). The index patient had one hyperplastic polyp removed from the rectum at the age of 43. Aged 44, she was diagnosed with bilateral endometrioid carcinoma of the ovaries with focally mucinous differentiation (Fig. 1), clinical stage 1B according to the FIGO staging system. Surgery was performed and she was treated with adjuvant chemotherapy comprising a regimen of cyclofosfamide and carboplatin. At age 45, 12.5 months later, she was diagnosed with an adenocarcinoma of the colon, treated by a left-sided hemicolectomy. Based on these clinical records the patient met the Amsterdam/Bethesda 

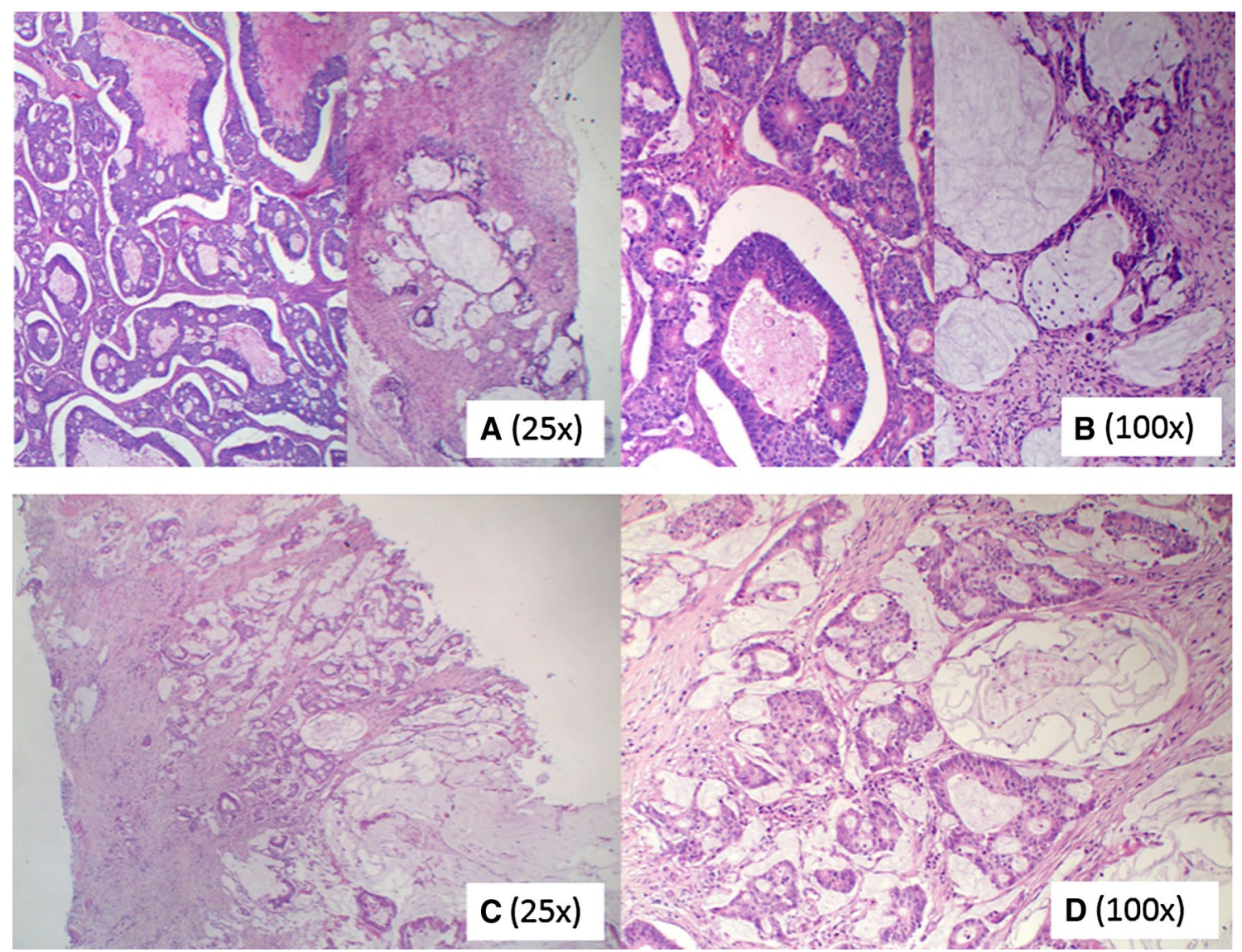

Fig. 1 Shows the histological picture of the ovarian tumor $(\mathbf{a}, \mathbf{b})$ and the colon tumor $(\mathbf{c}, \mathbf{d})$. In $\mathbf{a}, \mathbf{b}$ both endometrioid and mucinous parts of the ovarian tumor are shown

Table 1 Pathogenic variants, promoter methylation status and immunohistochemical expression of mismatch repair genes in the ovarian tumor, colon tumor and leukocyte DNA

\begin{tabular}{|c|c|c|c|}
\hline Gene & Ovary $(\mathrm{T} \%:>60 \%)$ & Colon (T\%: > 50\%) & Lymphocytes \\
\hline TP53 & No pathogenic variant & $\begin{array}{l}\text { c. } 1024 \mathrm{C}>\mathrm{T}, \mathrm{p} .(\operatorname{Arg} 342 *) / \\
6,1 \% \text { mutant reads }\end{array}$ & \\
\hline$M L H 1$ & $\begin{array}{l}\text { c. } 1624 \mathrm{C}>\mathrm{T}, \mathrm{p} .(\mathrm{Gln} 542 *) / \\
76 \% \text { mutant reads } \\
\text { LOH } \\
\text { Loss of expression by IHC } \\
\text { No promoter hypermethylation }\end{array}$ & $\begin{array}{l}\text { c. } 1624 \mathrm{C}>\mathrm{T}, \mathrm{p} .(\mathrm{Gln} 542 *) / \\
52 \% \text { mutant reads } \\
\text { LOH } \\
\text { Loss of expression by IHC } \\
\text { No promoter hypermethylation }\end{array}$ & No pathogenic variant \\
\hline CTNNB1 & $\begin{array}{l}\text { c. } 134 \mathrm{C}>\mathrm{T}, \mathrm{p} .(\text { Ser } 45 \mathrm{Phe}) / \\
9,2 \% \text { mutant reads }\end{array}$ & $\begin{array}{l}\text { c. } 134 \mathrm{C}>\mathrm{T}, \mathrm{p} .(\mathrm{Ser} 45 \mathrm{Phe}) \\
38 \% \text { mutant reads }\end{array}$ & \\
\hline$P M S 2$ & Loss of expression by IHC & Loss of expression by IHC & No pathogenic variant \\
\hline MSH2 & Normal expression by IHC & Normal expression by IHC & No pathogenic variant \\
\hline MSH6 & Normal expression by IHC & Normal expression by IHC & No pathogenic variant \\
\hline$A P C$ & No pathogenic variant & No pathogenic variant & \\
\hline
\end{tabular}

The table shows an overview of the detected pathogenic variants, methylation assays and immunohistochemical staining results of mismatch repair genes in one of the ovarian tumors, the colon tumor and DNA isolated from blood

$\mathrm{LOH}$ loss of heterozygosity, IHC immunohistochemical staining, $T \%$ tumor cell percentage 
revised criteria. Patient has remained disease-free until the age of 64 . However, the discovery of the (mucinous) colon carcinoma showing partly a similar morphology as the ovarian tumors (Fig. 1), created doubt about the primary origin of the ovarian tumors. Lynch syndrome (LS), in which independent ovarian and colon tumors had developed, was suggested.

Immunohistochemistry testing of the MMR proteins MLH1, PMS2, MSH2 and MSH6 of the colonic and ovarian tumors showed DNA MMR deficiency with loss of expression of MLH1 and PMS2. Microsatellite instability (MSI) testing using mononucleotide microsatellite markers showed an MSI-H phenotype. A sporadic origin of these MMR deficient tumors due to $\mathrm{MLHI}$ promoter hypermethylation was excluded. Our patient was subsequently referred to a clinical geneticist. However LS could not be confirmed after negative lymphocyte DNA testing of $M L H 1, P M S 2, M H S 2$ and $M S H 6$ for germline pathogenic variants. Also germline testing of $B R C A 1$ and $B R C A 2$ in two sisters of the patient was negative.

Reevaluation of the metachronously diagnosed colon tumor confirmed the primary origin in the colon as the bulk of the tumor was bulging in the colonic lumen. Furthermore, the serosal lining was unaffected. Immunohistochemical stainings of the ovarian tumor showed a phenotype compatible with a metastasis from a colon tumor (keratin-7 negative / keratine-20 and CDX-2 positive). ER, PR and vimentin were also negative. However, ovarian tumors with mucinous differentiation can show a wide variety of keratine-7/ keratin-20 immunoprofile patterns, and should be interpreted with caution [9].

Somatic testing (Table 1) of $M L H 1$ showed an identical $M L H 1$ class 5 pathogenic variant $(\mathrm{c} .1624 \mathrm{C}>\mathrm{T}$, p. $(\mathrm{Gln} 542 *))$ in both colon and ovarian tumors. Next, loss of heterozygosity for $M L H 1$ was shown by absence of the WT(wild type)-allele. We also somatically tested the complete $A P C$ gene for pathogenic variants in these lesions, as finding of pathogenic $A P C$ variants in ovarian neoplasms would point at a colonic origin of the lesions. No $A P C$ variants were found, however an identical activating class $5 C T N N B 1$ pathogenic variant $(\mathrm{c} .134 \mathrm{C}>\mathrm{T}$; p.(Ser45Phe)) was identified, the molecular alternative for Wnt-pathway activation (Fig. 2a). Finding identical $M L H 1$ and $C T N N B 1$ variants would suggest a clonal relation between the colon and ovarian tumor. Additionally, a class 5 pathogenic TP53 variant (c.1024C $>$ T,
Fig. 2 a Shows the reads including the pathogenic $C T N N B 1$ variant that is present in both the ovarian and the colon tumor. b Shows the reads including the pathogenic TP53 variant that is present in the colon tumor, but not in the ovarian tumor
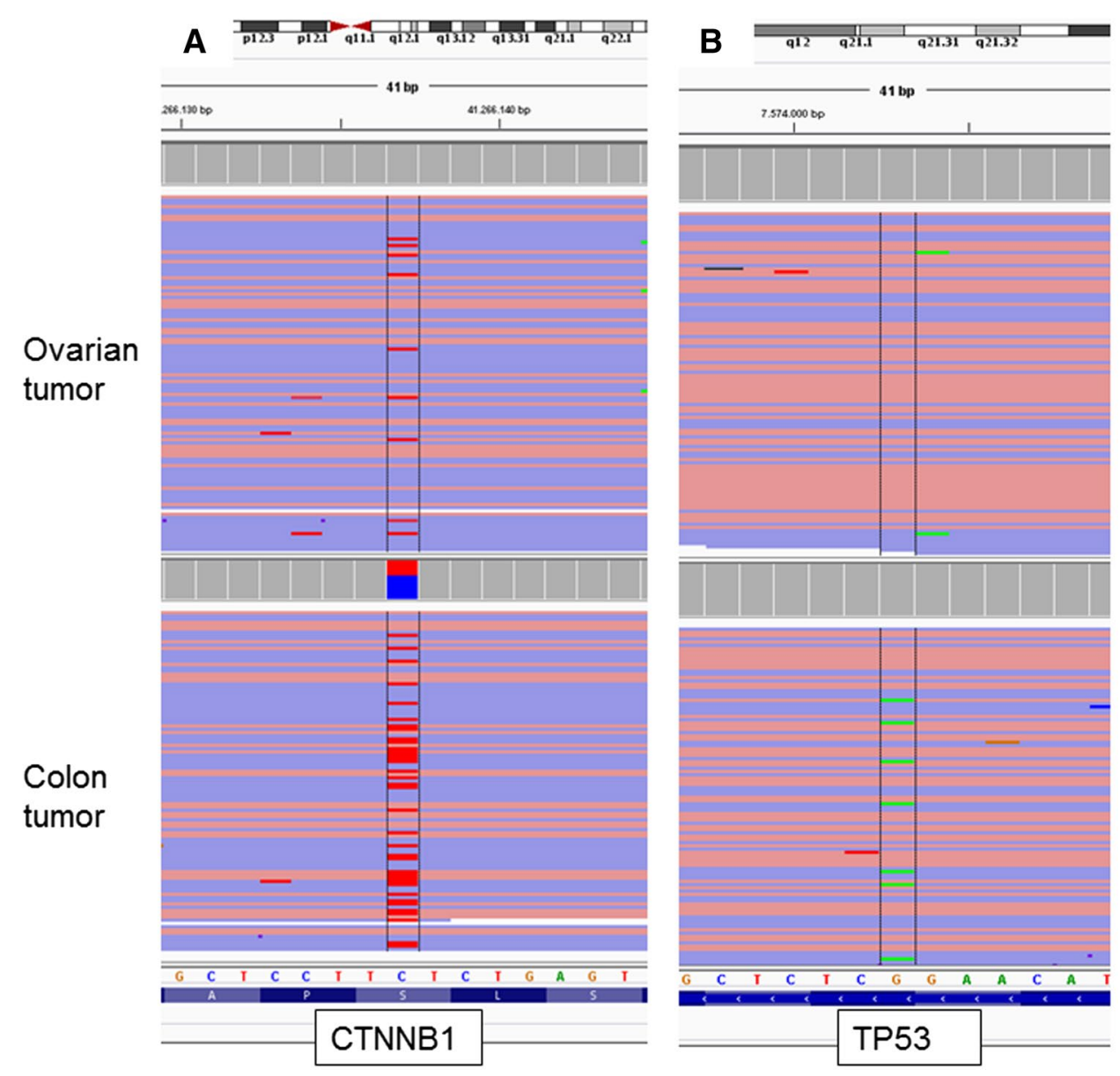
p. (Arg342*)) was detected in the colon tumor, but absent in the ovarian tumor (Fig. 2b). In order to estimate putative germline mosaicism we performed ultra-deep sequencing of the MLHI (c.1624C > T, p. (Gln542*)) variant in DNA isolated from normal colonic mucosa, saliva, blood and urine. All isolates showed sufficient ( $>10 \mathrm{~K}$ ) coverage, but showing no presence of the MLHI variant, rendering germline mosaicism unlikely. It was concluded that a metachronously diagnosed colorectal tumor that metastasized to both ovaries was the most likely diagnosis.

\section{Discussion}

In the current report we address a remarkable clinical dilemma once metachronous ovarian and colon tumors are diagnosed and the possibility of a Lynch syndrome needs to be answered. The female patient we now present with bilateral ovarian cancer was treated as having primary bilateral ovarian cancer. However, only 12.5 months after the first diagnosis the true primary origin of these lesions was questioned with the resection of a DNA mismatch repair deficient left sided colon cancer. After reevaluation and molecular analysis a clonal relation was identified between the ovarian and colonic lesions. As MMR deficient cancers mostly lack distant metastases possibly due to the interaction with the immune system, it is noteworthy that this DNA MMR deficient colon cancer probably metastasized to the ovaries [10].

About $15 \%$ of all ovarian tumors turn out to be metastases [2]. Histological parameters are not always sufficient to discriminate between a primary tumor and/or metastasis. Nowadays, molecular analysis can be a helpful tool to make this distinction in selected cases [11]. Inactivating $A P C$ pathogenic variants are almost exclusively found in colon tumors. Thus, the presence of a pathogenic variant is a strong argument for a primary colon tumor $[3,11,12]$. In our patient no pathogenic variants in the $A P C$ gene were found, but an identical activating $C T N N B 1$ variant was present in both ovary and colon tumors. As $C T N N B 1$ variants are very rare in colon carcinomas, this might suggest the ovarian tumor as the primary origin [13]. On the other hand activating CTNNB1 pathogenic variants are often found in colon cancer associated with DNA mismatch repair deficiency [14-16]. Only an incidental report of metastatic mismatch repair deficient colon carcinoma to the ovaries is described [17]. In previous published research we did not find any CTNNB1 pathogenic variants in MMR proficient colorectal metastases to the ovary [11]. With respect to the ovary, CTNNB1 pathogenic variants have mainly been found in endometrioid ovarian cancers [13]. However, the histopathological findings in our case do not suggest metastases from the ovary since the colonic tumor was located at the luminal site. In case of a metastasis the bulk of the tumor would have been present on the serosal site. Besides, ovarian cancers metastasizing to the colon, and morphologically mimicking a primary colon tumor are probably very rare. Furthermore in case of bilateral ovarian tumors the odds favor metastases from a primary tumor elsewhere in the body.

In our patient the same somatic $M L H 1$ pathogenic variant and concomitant loss of heterozygosity of the wild type allele was present in the ovarian and colon cancer. As the detected $\mathrm{MLHI}$ variant was not found by deep sequencing of DNA isolated from normal mucosa, saliva, blood and urine a germline mosaicism was rendered unlikely.

Somatic $M L H 1$ pathogenic variants in sporadic tumors are mainly associated with gastrointestinal tumors [13, 18]. $M L H 1$ pathogenic variants are not commonly found in ovarian cancer, although one study found $M L H I$ pathogenic variants in $8.7 \%$ epithelial ovarian cancer [19]. Usually, TP53 pathogenic variants occur early in the evolutionary development of a tumor. Our patient's tumors showed in two tumors $C T N N B 1$ as well as $M L H 1$ pathogenic variants, but only in the colon tumor a TP53 pathogenic variant was identified. The presence of this variant can be explained by tumor progression within the primary colon tumor. Apparently in this case, the pathogenic TP53 variant is not present in the metastasizing clone. Such spatial differences in mutation profiles within a tumor are known as intra-tumor heterogeneity.

In summary, we discuss the clinical dilemma with metachronous diagnosed bilateral mismatch repair deficient ovarian and colon cancer harboring a pathogenic MMR variant. In our case Lynch syndrome as well as a postzygotic somatic mutation leading to mosaicism of multiple normal tissues are very unlikely. Molecular analysis showed a clonal relationship between the ovarian and colon tumors with histopathological analysis suggesting the colon tumor being the primary tumor.

Acknowledgements We thank M.A. Wijngaarden for help with writing and critically reading the manuscript.

\section{Compliance with ethical standards}

Conflict of interest The authors declare that they have no conflict of interests.

Open Access This article is distributed under the terms of the Creative Commons Attribution 4.0 International License (http://creativecommons.org/licenses/by/4.0/), which permits unrestricted use, distribution, and reproduction in any medium, provided you give appropriate credit to the original author(s) and the source, provide a link to the Creative Commons license, and indicate if changes were made. 


\section{References}

1. Khunamornpong S et al (2006) Primary and metastatic mucinous adenocarcinomas of the ovary: evaluation of the diagnostic approach using tumor size and laterality. Gynecol Oncol 101(1):152-157

2. de Waal YR et al (2009) Secondary ovarian malignancies: frequency, origin, and characteristics. Int J Gynecol Cancer 19(7):1160-1165

3. Kelemen LE, Kobel M (2011) Mucinous carcinomas of the ovary and colorectum: different organ, same dilemma. Lancet Oncol 12(11):1071-1080

4. Lee KR, Young RH (2003) The distinction between primary and metastatic mucinous carcinomas of the ovary: gross and histologic findings in 50 cases. Am J Surg Pathol 27(3):281-292

5. Lips EH et al (2008) Progression and tumor heterogeneity analysis in early rectal cancer. Clin Cancer Res 14(3):772-781

6. Homig-Holzel C, Savola S (2012) Multiplex ligation-dependent probe amplification (MLPA) in tumor diagnostics and prognostics. Diagn Mol Pathol 21(4):189-206

7. Boland CR et al (1998) A National Cancer Institute Workshop on Microsatellite Instability for cancer detection and familial predisposition: development of international criteria for the determination of microsatellite instability in colorectal cancer. Cancer Res 58(22):5248-5257

8. Jansen AM et al (2017) Distinct patterns of somatic mosaicism in the apc gene in neoplasms from patients with unexplained adenomatous polyposis. Gastroenterology 152(3):546-549

9. Cathro HP, Stoler MH (2002) Expression of cytokeratins 7 and 20 in ovarian neoplasia. Am J Clin Pathol 117(6):944-951

10. de Miranda NF et al (2012) Role of the microenvironment in the tumourigenesis of microsatellite unstable and MUTYH-associated polyposis colorectal cancers. Mutagenesis 27(2):247-253
11. Crobach S et al (2015) Target-enriched next-generation sequencing reveals differences between primary and secondary ovarian tumors in formalin-fixed, paraffin-embedded tissue. J Mol Diagn 17(2):193-200

12. Miyoshi Y et al (1992) Somatic mutations of the APC gene in colorectal tumors: mutation cluster region in the APC gene. Hum Mol Genet 1(4):229-233

13. Bamford S et al (2004) The COSMIC (catalogue of somatic mutations in cancer) database and website. Br J Cancer 91(2):355-358

14. Lovig T et al (2002) APC and CTNNB1 mutations in a large series of sporadic colorectal carcinomas stratified by the microsatellite instability status. Scand J Gastroenterol 37(10):1184-1193

15. Johnson V et al (2005) Exon 3 beta-catenin mutations are specifically associated with colorectal carcinomas in hereditary nonpolyposis colorectal cancer syndrome. Gut 54(2):264-267

16. Mirabelli-Primdahl L et al (1999) Beta-catenin mutations are specific for colorectal carcinomas with microsatellite instability but occur in endometrial carcinomas irrespective of mutator pathway. Cancer Res 59(14):3346-3351

17. Ongom PA et al (2013) Metastatic colorectal carcinoma mimicking primary ovarian carcinoma presenting as 'giant' ovarian tumors in an individual with probable Lynch syndrome: a case report. J Med Case Rep 7:158

18. Herfarth KK et al (1997) Mutations in MLH1 are more frequent than in MSH2 in sporadic colorectal cancers with microsatellite instability. Genes Chromosomes Cancer 18(1):42-49

19. Kim YM et al (2014) Analysis and comparison of somatic mutations in paired primary and recurrent epithelial ovarian cancer samples. PLoS ONE 9(6):e99451 